\title{
Stability and Convergence of Difference Approximations to Pseudo-Parabolic Partial Differential Equations
}

\author{
By William H. Ford* and T. W. Ting**
}

\begin{abstract}
Two difference approximations to the solution of a pseudo-parabolic problem are constructed and shown by means of stability analysis to converge in the "discrete" $L_{2}$ norm. A relation between parabolic and pseudo-parabolic difference schemes is discussed, and the stability of difference approximations to backward time parabolic and pseudo-parabolic problems is also considered.
\end{abstract}

1. Introduction. We shall let $\bar{R}=\{(t, x): 0 \leqq t \leqq T, 0 \leqq x \leqq 1\}$ and consider difference approximations to the strict solution $u(t, x)$ of the linear problem,

$$
\begin{gathered}
\left(a(t, x) u_{t x}\right)_{x}+\left(b(t, x) u_{x}\right)_{x}-q(t, x) u=r(t, x) u_{t} \quad \text { in } \bar{R}, \\
u(0, x)=u_{0}(x), \quad 0 \leqq x \leqq 1, \\
u(t, 0)=f(t), \quad u(t, 1)=g(t), \quad 0 \leqq t \leqq T .
\end{gathered}
$$

Actually, with slight modifications our methods are also applicable to semilinear equations. Throughout this paper, we assume that (i) the given functions $a, b, r$, and $q$ belong to the class $C^{3}(\bar{R})$; (ii) there are positive constants $A_{*}, R_{*}$ such that $a(t, x) \geqq$ $A_{*}, r(t, x) \geqq R_{*}$ in $\bar{R}$; (iii) problem (1.1) has a unique solution $u$ in $C^{5}(\bar{R})$. In passing, we note that in $\bar{R},|b(t, x)| \leqq B^{*},|q(t, x)| \leqq Q^{*}$, where $B^{*}, Q^{*}$ are constants.

We formulate two difference approximations to the solution of (1.1), apply a stability analysis method developed by Douglas [4, pp. 41-44] to establish the convergence of each approximation in the "discrete" $L_{2}$ norm, and prove that for a fixed lattice, the solution to each of two difference approximations to a certain parabolic problem can be obtained as the limit of a sequence of difference approximations to a pseudo-parabolic problem. Lastly, a comparison of the stability properties of difference schemes is made for backward time linear parabolic and pseudo-parabolic problems.

Various physical applications of pseudo-parabolic equations are discussed in [13], [12], [7]. The most significant application of all is to the two-temperature theory of heat conduction proposed by Chen and Gurtin [1].

2. Stability of the Difference Schemes. Let $k=T / M, h=1 / N$, and take $t_{n}=n k, 0 \leqq n \leqq M, x_{\alpha}=\alpha h, 0 \leqq \alpha \leqq N$, so that the points $\left\{\left(t_{n}, x_{\alpha}\right)\right\}$ form a lattice covering $\bar{R}$. The difference notation used is standard, and the reader may consult [4, pp. 2-4].

Received July 26, 1972, revised January 22, 1973.

AMS (MOS) subject classifications (1970). Primary 65M10.

Key words and phrases. Stability, difference schemes, pseudo-parabolic equations.

* The work of this author was partially supported by an NDEA Title IV Fellowship.

** The work of this author was partially supported by NSF Grant GP-33174X. 
Choose $j$ to be either 0 or $\frac{1}{2}$ and define the linear operators $\Delta_{n+j}, \Gamma_{n+i}$ on $R^{N-1}$ such that, for $1 \leqq \alpha \leqq N-1$,

$$
\begin{array}{ll}
\left(\Delta_{n+j} \phi\right)_{\alpha}=r_{n+i, \alpha} \phi_{\alpha}-\Delta_{x}\left(a_{n+i} \Delta_{x} \phi\right)_{\alpha}, & \phi_{0}=\phi_{N}=0, \\
\left(\Gamma_{n+j} \phi\right)_{\alpha}=q_{n+i, \alpha} \phi_{\alpha}-\Delta_{x}\left(b_{n+j} \Delta_{x} \phi\right)_{\alpha}, & \phi_{0}=\phi_{N}=0,
\end{array}
$$

for all vectors $\phi=\left(\phi_{1}, \cdots, \phi_{N-1}\right)$, where $r, a, b, q$ are functions appearing in (1.1). Let $A_{n+i}, B_{n+i}$ be, respectively, the matrices of $\Lambda_{n+i}$ and $\Gamma_{n+i}$ relative to the standard ordered basis for $R^{N-1}$. We shall consider the following two difference schemes, which we call the standard and Crank-Nicolson, respectively, for the approximation of the solution to (1.1):

$$
\begin{gathered}
k^{-1} A_{n} w_{n+1}=\left(k^{-1} A_{n}-B_{n}\right) w_{n}+c_{n}, \quad w_{0}=u_{0}, \\
\left(k^{-1} A_{n+1 / 2}+\frac{1}{2} B_{n+1 / 2}\right) w_{n+1}=\left(k^{-1} A_{n+1 / 2}-\frac{1}{2} B_{n+1 / 2}\right) w_{n}+d_{n}, \quad w_{0}=u_{0},
\end{gathered}
$$

where, for $0 \leqq n \leqq M-1$,

$$
\begin{array}{r}
c_{n \alpha}=\left\{\begin{array}{lr}
h^{-2} k^{-1} a_{n, 1 / 2}\left(f\left(t_{n}\right)-f\left(t_{n+1}\right)\right)-h^{-2} b_{n, 1 / 2} f\left(t_{n}\right), & \alpha=1, \\
0, & 2 \leqq \alpha \leqq N-2, \\
h^{-2} k^{-1} a_{n, N-1 / 2}\left(g\left(t_{n}\right)-g\left(t_{n+1}\right)\right)-h^{-2} b_{n, N-1 / 2} g\left(t_{n}\right), & \alpha=N-1,
\end{array}\right. \\
d_{n \alpha}=\left\{\begin{array}{lr}
h^{-2} k^{-1} a_{n+1 / 2,1 / 2}\left(f\left(t_{n}\right)-f\left(t_{n+1}\right)\right)-\frac{1}{2} h^{-2} b_{n+1 / 2,1 / 2}\left(f\left(t_{n+1}\right)+f\left(t_{n}\right)\right), \quad \alpha=1, \\
0, & 2 \leqq \alpha \leqq N-2, \\
h^{-2} k^{-1} a_{n+1 / 2, N-1 / 2}\left(g\left(t_{n}\right)-g\left(t_{n+1}\right)\right)-\frac{1}{2} h^{-2} b_{n+1 / 2, N-1 / 2}\left(g\left(t_{n+1}\right)+g\left(t_{n}\right)\right), & \alpha=N-1,
\end{array}\right.
\end{array}
$$

$f$ and $g$ are the boundary values in (1.1), and $u_{0}$ is the vector containing the initial condition.

The tridiagonal matrices involved in the schemes are such that (2.1) has a unique solution for all $k$, and (2.2) has a unique solution for all sufficiently small $k$.

Our stability and convergence analysis will be done for the inner product $(\phi, \psi)^{\prime}=$ $h \sum_{\alpha=1}^{N-1} \phi_{\alpha} \psi_{\alpha}$ and induced norm $\|\phi\|=\left[h \sum_{\alpha=1}^{N-1}\left(\phi_{\alpha}\right)^{2}\right]^{1 / 2}$, which we call the $L_{2}$ norm. It is seen from their definition that the matrices $A_{n+j}, B_{n+i}$ are symmetric for $j=0$ or $\frac{1}{2}$ and for all $n$, and simple calculation shows that

$$
\left(A_{n+i} \phi, \phi\right)=h \sum_{\alpha=1}^{N}\left\{a_{n+i, \alpha-1 / 2}\left[\left(\phi_{\alpha}-\phi_{\alpha-1}\right) / h\right]^{2}+r_{n+i, \alpha}\left(\phi_{\alpha}\right)^{2}\right\},
$$

where $\phi_{0}=\phi_{N}=0$. A similar relation holds for $B_{n+j}$. Now, (2.3) and assumption (ii) ensure that the matrices $A_{n+i}$ are positive definite, and so we may define the sequence of inner products $(\phi, \psi)_{n+i}=\left(A_{n+i} \phi, \psi\right)$ and induced norms $\|\phi\|_{n+i}=\left(A_{n+j} \phi, \phi\right)^{1 / 2}$ on $R^{N-1}$. We may now define stability for the schemes (2.1), (2.2).

Definition 2.1. The difference scheme (2.1) [(2.2)] is said to be stable if there exists a positive constant $s$, depending on the structure of problem (1.1) and independent of the time level $n$ and time increment $k$, such that whenever $\phi_{n}$ and $\phi_{n+1}$ are any two vectors in $R^{N-1}$ satisfying the homogeneous system corresponding to (2.1) [(2.2)] at time level $n$, then $\left\|\phi_{n+1}\right\|_{n+i} \leqq(1+s k)\left\|\phi_{n}\right\|_{n+i}$ with $j=0$ [with $j=\frac{1}{2}$ ], for all $n$. 
To prove the stability of the schemes, we follow Douglas' approach in [3] and reduce the problem to the estimation of bounds on the eigenvalues of a generalized eigenvalue problem of the form

$$
B_{n+i} \phi=\mu A_{n+i} \phi .
$$

Note that there exists a complete set of eigenvectors for (2.4), with corresponding real eigenvalues, orthonormal with respect to the inner product $(\cdot, \cdot)_{n+i}[2, \mathrm{pp} .37-41]$, and if we apply the minimax principle [7, p. 181], it is easily seen by using (2.3) and the similar relation for $B_{n+i}$ that the least eigenvalue of (2.4) is bounded below by the least eigenvalue for the eigenvalue problem

$$
-\left(Q^{*} \phi_{\alpha}-B^{*} \Delta_{x}^{2} \phi_{\alpha}\right)=\nu\left(R_{*} \phi_{\alpha}-A_{*} \Delta_{x}^{2} \phi_{\alpha}\right), \quad 1 \leqq \alpha \leqq N-1,
$$

where $\phi_{0}=\phi_{N}=0$. The eigenvalues of $(2.5)$ can be calculated exactly [4, p. 4], and a simple assumption on $Q^{*}$ yields a lower bound for the least eigenvalue of (2.4). A similar argument provides a bound for the largest eigenvalue. In fact, there follows

LEMMA 2.2. Let $\mu_{n}^{(1)}$ and $\mu_{n}^{(N-1)}$ be, respectively, the least and greatest eigenvalues of (2.4). Let $Q^{*}$ be increased, if necessary, so that $B^{*} R_{*}-A_{*} Q^{*} \leqq 0$. Then $\mu_{n}^{(1)} \geqq$ $-Q^{*} / R_{*}$ and $\mu_{n}^{(N-1)} \leqq Q^{*} / R_{*}$.

Throughout the remainder of this paper, when $Q^{*}$ is needed, it will be assumed that $Q^{*}$ is chosen to satisfy this requirement.

We are now in a position to prove that each of the two schemes is stable.

THEOREM 2.3. For all time increments $k$, the standard scheme (2.1) is stable.

Proof. If we apply Lemma 2.2 to the eigenvalue problem $\left(k^{-1} A_{n}-B_{n}\right) \phi=\lambda k^{-1} A_{n} \phi$, it follows that $\max \left|\lambda_{n}^{(\alpha)}\right| \leqq 1+k Q^{*} / R_{*}$. There exists a complete orthonormal set of eigenvectors $\phi_{n}^{(1)}, \cdots, \phi_{n}^{(N-1)}$ for this problem, and so if $\phi_{n}=\sum_{\alpha=1}^{N-1} C_{n \alpha} \phi_{n}^{(\alpha)}, \phi_{n+1}=$ $\sum_{\alpha=1}^{N-1} D_{n \alpha} \phi_{n}^{(\alpha)}$ are any two vectors such that $k^{-1} A_{n} \phi_{n+1}=\left(k^{-1} A_{n}-B_{n}\right) \phi_{n}$, it follows that $D_{n \alpha}=\lambda_{n}^{(\alpha)} C_{n \alpha}, 1 \leqq \alpha \leqq N-1$. The orthonormality of the $\phi_{n}^{(\alpha)}$ then implies that $\left\|\phi_{n+1}\right\|_{n} \leqq\left(1+k Q^{*} / R_{*}\right)\left\|\phi_{n}\right\|_{n}$.

THEOREM 2.4. If $\epsilon$ is fixed, $0<\epsilon<1$, and the time increment $k$ is chosen so small that $k Q^{*} /\left(2 R_{*}\right) \leqq 1-\epsilon$, then the Crank-Nicolson scheme (2.2) is stable.

Proof. If we apply Lemma 2.2 to the eigenvalue problem $\left(k^{-1} A_{n+1 / 2}-\frac{1}{2} B_{n+1 / 2}\right) \phi=$ $\lambda\left(k^{-1} A_{n+1 / 2}+\frac{1}{2} B_{n+1 / 2}\right) \phi$, it follows that $\left(1-\lambda_{n}^{(\alpha)}\right) /\left(1+\lambda_{n}^{(\alpha)}\right) \geqq-k Q^{*} /\left(2 R_{*}\right)$, and the assumption on $k$ implies that $-1<\lambda_{n}^{(\alpha)} \leqq 1+k Q^{*} /\left(\epsilon R_{*}\right)$. The rest of the proof proceeds as in Theorem 2.3.

3. Convergence of the Difference Approximations. In this section, the stability of the difference schemes will be used to demonstrate $L_{2}$ convergence of the approximations as the lattice size diminishes.

THEOREM 3.1. Under assumptions (i)-(iii) as stated in the Introduction, the solution of the standard difference approximation (2.1) to problem (1.1) converges in the "discrete" $L_{2}$ norm as the lattice size diminishes to zero. Moreover, the rate of convergence is $O\left(h^{2}+k\right)$.

Proof. It is an immediate consequence of assumption (i) that there exists a constant $t$, independent of $n$ and $k$ such that, for all $\phi$,

$$
\|\phi\|_{n+1} \leqq(1+t k)\|\phi\|_{n} \text { for all } n .
$$

From (2.3) and assumption (ii) it follows that, for all $\phi$, 


$$
\|\phi\| \leqq\|\phi\|_{n} /\left(R_{*}\right)^{1 / 2} \text { for all } n .
$$

If we let $z=u-w$, where $w$ is the solution of (2.1), it is readily seen by Taylor's theorem that the error $z$ satisfies the difference system $k^{-1} A_{n} z_{n+1}=\left(k^{-1} A_{n}-B_{n}\right) z_{n}+$ $e_{n}, z_{0}=0$, where $e_{n \alpha}=O\left(h^{2}+k\right), 1 \leqq \alpha \leqq N-1$. A direct calculation shows that $k^{-2}\left\|\left(k^{-1} A_{n}\right)^{-1} e_{n}\right\|_{n}^{2} \leqq\left\|A_{n}^{-1}\right\|\left\|e_{n}\right\|^{2}$, and since $\left\|A_{n}^{-1}\right\| \leqq R_{*}^{-1}$ and $\left\|e_{n}\right\|=O\left(h^{2}+k\right)$ for all $n$, it follows that

$$
k^{-1}\left\|\left(k^{-1} A_{n}\right)^{-1} e_{n}\right\|_{n}=O\left(h^{2}+k\right) \text { as } h, k \rightarrow 0 .
$$

The stability of scheme (2.1) coupled with (3.1)-(3.3) are shown in [4, pp. 41-44] to imply convergence in $L_{2}$.

We shall now consider the Crank-Nicolson approximation (2.2). Before proving convergence, a preliminary lemma will be stated without proof. Recall that the spectral radius $\zeta(C)$ of an $m \times m$ matrix $C$ is given by $\zeta(C)=\max \left|\lambda_{i}\right|$, where $\lambda_{i}, 1 \leqq i \leqq m$, are the eigenvalues of $C$.

Lemma 3.2. Let $A$ be a real $m \times m$ symmetric positive definite matrix. Let $\|\phi\|_{A}=$ $(A \phi, \phi)^{1 / 2}, \phi \in R^{m}$. Then, for every $m \times m$ matrix $B$,

$$
\|B\|_{A}=\left(\zeta\left(A^{-1} B^{*} A B\right)\right)^{1 / 2},
$$

where $B^{*}$ is the adjoint of $B$.

THEOREM 3.3. Under the stated restrictions on $u, a, b, q$, and $r$ indicated in (i)-(iii), the solution of the Crank-Nicolson approximation (2.2) to problem (1.1) converges in the "discrete" $L_{2}$ norm as the mesh size decreases to zero. Moreover, the rate of convergence is $O\left(h^{2}+k^{2}\right)$.

Proof. Relations (3.1) and (3.2) follow for the norm $\|\cdot\|_{n+1 / 2}$, and the scheme (2.2) is stable for sufficiently small $k$. The error equation is $\left(k^{-1} A_{n+1 / 2}+\frac{1}{2} B_{n+1 / 2}\right) z_{n+1}=$ $\left(k^{-1} A_{n+1 / 2}-\frac{1}{2} B_{n+1 / 2}\right) z_{n}+e_{n}, z_{0}=0$, where $e_{n \alpha}=O\left(h^{2}+k^{2}\right), 1 \leqq \alpha \leqq N-1$, and so by [4, pp. 41-44] all we need do to prove convergence is to show that $k^{-1}\left\|C_{n+1 / 2}^{-1} e_{n}\right\|_{n+1 / 2}=O\left(h^{2}+k^{2}\right)$, where $C_{n+1 / 2}=k^{-1} A_{n+1 / 2}+\frac{1}{2} B_{n+1 / 2}$. A straightforward computation using the symmetry of $C_{n+1 / 2}$ and $A_{n+1 / 2}$ shows that

$$
\begin{aligned}
\left\|C_{n+1 / 2}^{-1} e_{n}\right\|_{n+1 / 2}^{2} & \leqq\left\|C_{n+1 / 2}^{-1} A_{n+1 / 2} C_{n+1 / 2}^{-1}\right\|\left\|e_{n}\right\|^{2} \\
& \leqq\left\|C_{n+1 / 2}^{-1} A_{n+1 / 2}\right\|_{n+1 / 2}\left\|C_{n+1 / 2}^{-1}\right\|_{n+1 / 2}\left\|e_{n}\right\|^{2} .
\end{aligned}
$$

Consider the eigenvalue problem $A_{n+1 / 2} \phi=\lambda C_{n+1 / 2} \phi$. By using Lemma 2.2 and the restriction on $k$ given in Theorem 2.4 , it follows that $0<\lambda \leqq k / \epsilon$ and subsequently that $\left\|C_{n+1 / 2}^{-1} A_{n+1 / 2}\right\|_{n+1 / 2}=O(k)$ as $k \rightarrow 0$. By Lemma 3.2 and the symmetry of $C_{n+1 / 2}$,

$$
\left\|C_{n+1 / 2}^{-1}\right\|_{n+1 / 2}^{2} \leqq\left\|C_{n+1 / 2}^{-1} A_{n+1 / 2}||_{n+1 / 2}\right\| A_{n+1 / 2}^{-1}\left\|_{n+1 / 2}\right\| C_{n+1 / 2}^{-1} \|_{n+1 / 2}
$$

and so

$$
|| C_{n+1 / 2}^{-1}\left\|_{n+1 / 2} \leqq\right\| C_{n+1 / 2}^{-1} A_{n+1 / 2}||_{n+1 / 2}\left\|A_{n+1 / 2}^{-1}\right\|_{n+1 / 2} .
$$

Since $\left\|A_{n+1 / 2}^{-1}\right\|_{n+1 / 2} \leqq\left(R_{*}\right)^{-1}$ by Lemma 3.2, there results $\left\|C_{n+1 / 2}^{-1}\right\|_{n+1 / 2}=O(k)$. If we note that $\left\|e_{n}\right\|=O\left(h^{2}+k^{2}\right)$ and use (3.4), the theorem follows.

We now briefly discuss difference approximations to the semilinear problem

$$
\left(a(t, x) u_{t x}\right)_{x}+\left(b(t, x) u_{x}\right)_{x}-q(t, x, u)=r(t, x) u_{t} \text { in } \bar{R},
$$

with the same initial-boundary values as in (1.1). We assume that $u, a, b, r$ have the 
properties listed in (i)-(iii). The function $q$ is assumed to have a bounded derivative in $u$ for all $(t, x) \in \bar{R},-\infty<u<\infty$. The corresponding standard scheme takes on the same form as in (2.1) except the terms involving $q_{n \alpha} w_{n \alpha}$ are replaced by $q\left(t_{n}, x_{\alpha}, w_{n \alpha}\right)$. Similarly, the Crank-Nicolson scheme has the same form as (2.2) except that the terms involving $q$ are replaced by $-q\left(t_{n+1 / 2}, x_{\alpha},\left(w_{n+1, \alpha}+w_{n \alpha}\right) / 2\right)$. The standard difference system is still linear, while that of the Crank-Nicolson scheme is nonlinear and must be solved by an iterative process. The error equation for each scheme is readily seen to be of precisely the same form as those in the last section, and those techniques prove that the standard scheme converges with rate $O\left(h^{2}+k\right)$ and the Crank-Nicolson with rate $O\left(h^{2}+k^{2}\right)$.

4. Parabolic and Pseudo-Parabolic Difference Schemes. In this section, we shall discuss a relation between parabolic and pseudo-parabolic equations. Consider the linear parabolic problem

$$
\left(b(t, x) u_{x}\right)_{x}-q(t, x) u=r(t, x) u_{t} \quad \text { in } R,
$$

with the same initial-boundary conditions as in (1.1), and its explicit difference approximation

$$
\begin{gathered}
\Delta_{x}\left(b_{n} \Delta_{x} p_{n}\right)_{\alpha}-q_{n \alpha} p_{n \alpha}=r_{n \alpha}\left(p_{n+1, \alpha}-p_{n \alpha}\right) / k, \quad 1 \leqq \alpha \leqq N-1, \\
p_{0 \alpha}=u_{0}\left(x_{\alpha}\right), \quad p_{n+1,0}=f\left(t_{n+1}\right), \quad p_{n+1, N}=g\left(t_{n+1}\right) .
\end{gathered}
$$

Let $\epsilon$ be a positive parameter. We shall relate the difference approximation (4.2) to the standard scheme for the pseudo-parabolic problem

$$
\epsilon\left(a(t, x) u_{t x}^{\epsilon}\right)_{x}+\left(b(t, x) u_{x}^{\epsilon}\right)_{x}-q(t, x) u^{\epsilon}=r(t, x) u_{t}^{\epsilon} \quad \text { in } \bar{R},
$$

with the same initial-boundary conditions as in (1.1). Assumptions (i)-(ii) are to hold for $a, b, r, q$, but in addition it is assumed that $0<B_{*} \leqq b(t, x) \leqq B^{*}$ in $\bar{R}$. It is known [14] that under suitable regularity conditions, the solution $u^{\epsilon}$ of (4.3) converges, for every value of $t$, to the solution $u$ of $(4.1)$ in $L_{2}[0,1]$ as $\epsilon \rightarrow 0$. We have a corresponding result here for difference schemes. The proof proceeds by subtracting the standard scheme for (4.3) with solution $w^{\epsilon}$ from (4.2) and observing that $\left(p-w^{\epsilon}\right)$ satisfies a nonhomogeneous parabolic difference scheme, with zero boundary and initial values, whose nonhomogeneous term depends on $w^{\epsilon}$ and $\epsilon$. If it is shown that $\max \left|w_{n \alpha}^{e}\right|$ is bounded independent of $\epsilon$, then the results of [3] provide the result. To show this, an eigenvalue analysis is performed on the difference equation satisfied by $w^{\epsilon}$, and the result follows by iteration along the lines of [4, pp. 41-44]. Details are available from either of the authors.

THEOREM 4.1. Let the mesh ratio $\lambda=k / h^{2}$ be such that $\lambda \leqq R_{*} /\left(2 B^{*}\right)$. Let $p$ be the solution to (4.2) and $w^{\epsilon}$ the solution of the standard approximation to (4.3). Then, for $0 \leqq n \leqq M,\left\|p_{n}-w_{n}^{e}\right\|=O(\epsilon)$ as $\epsilon \rightarrow 0$.

The same type of result can be proved for the Crank-Nicolson approximation. Of course, in this case we have to use the Crank-Nicolson difference approximations for both (4.1) and (4.3). In fact, we have

THEOREM 4.2. Let $p$ and $w^{\epsilon}$ be, respectively, the Crank-Nicolson approximations to (4.1) and (4.3). Then, for all $0 \leqq n \leqq M,\left\|p_{n}-w_{n}^{\epsilon}\right\|=O(\epsilon)$ as $\epsilon \rightarrow 0$. 
It is well known that the explicit difference formulation for the parabolic problem (4.1) cannot be expected to be stable for $\lambda>R_{*} /\left(2 B^{*}\right)$, so the restricted mesh ratio is not surprising. On the other hand, the Crank-Nicolson approximation to (4.1) is unconditionally stable, so no restriction on the mesh ratio is expected in this case.

5. Stability for the Backward Time Problem. To conclude our discussion, we briefly mention the backward time problem in the parabolic and pseudo-parabolic case. It is well known that for parabolic differential equations there is, in general, no solution. For pseudo-parabolic equations, on the other hand, with sufficient regularity conditions on the coefficients and the initial and boundary data, a unique solution exists for $-\infty<t<\infty$ [12]. An analogous situation exists in the discrete case. The standard approximation to (1.1) written out for negative time remains stable. In fact, the eigenvalue problem considered for the determination of stability is $B_{n} \phi=$ $(\lambda-1) k^{-1} A_{n} \phi$, the only difference being that here $(\lambda-1) k^{-1}$ multiplies $A_{n} \phi$, whereas in the nonnegative time problem $(1-\lambda) k^{-1}$ multiplies $A_{n} \phi$. Exactly as before, we have $\max _{\alpha}\left|\lambda_{\alpha}\right| \leqq 1+Q^{*} k / R_{*}$. The eigenvalue problem for the determination of stability for the backward time explicit difference scheme for the corresponding parabolic problem (4.1) is $B_{n} \phi=(\lambda-1) k^{-1} R_{n} \phi$, where $R_{n \alpha}=r_{n \alpha}, 1 \leqq \alpha \leqq N-1$. The best we can say in this case is that $\lambda \leqq 1+k Q^{*} / R_{*}+\left(4 B^{*} / R_{*}\right)\left(k / h^{2}\right)$, so we cannot expect stability.

The Crank-Nicolson approximation to (1.1) in the backward time case is still stable for sufficiently small $k$, while we cannot expect stability for the corresponding parabolic problem.

Department of Mathematical Sciences

Clemson University

Clemson, South Carolina 29631

Mathematics Department

University of Illinois

Urbana, Illinois 61801

1. P. J. Chen \& M. E. Gurtin, "On a theory of heat conduction involving two temperatures,"J. Appl. Math Phys., v. 19, 1968, pp. 614-627.

2. R. Courant \& D. Hilbert, Methoden der Mathematischen Physik. Vol. I, Springer, Berlin, 1931; English transl., Interscience, New York, 1953. MR 16, 426.

3. J. Douglas, JR., "The application of stability analysis in the numerical solution of quasi-linear parabolic differential equations," Trans. Amer. Math. Soc., v. 89, 1958, pp. 484518. MR 24 \#A1521.

4. J. Douglas, JR., A Survey of Numerical Methods for Parabolic Differential Equations, Advances in Computers, vol. II, Academic Press, New York, 1961, pp. 1-54. MR 25 \# 5604. 5. W. H. FORD \& T. W. TING, "Uniform error estimates for difference approximations to non-linear pseudo-parabolic partial differential equations," SIAM J. Numer. Anal. (To appear.)

6. G. FORSYTHE \& C. B. Moler, Computer Solutions of Linear Algebraic Systems, Prentice-Hall, Englewood Cliffs, N.J., 1967. MR 36 \#2306.

7. H. GAJEWSKI \& K. ZACHARIAS, "Zur starken Konvergenz des Galerkinverfahrens bei einer Klasse pseudoparabolischer partieller Differentialgleichungen," Math. Nachr., v. 47, 1970, pp. 365-376. MR 44 \#4351.

8. P. R. Halmos, Finite Dimensional Vector Spaces, 2nd ed., Van Nostrand, Princeton, N.J., 1958. MR 19, 725 .

9. E. IsAacson \& H. B. Keller, Analysis of Numerical Methods, Wiley, New York, 1966. MR 34 \#924.

10. F. JoHN, "On integration of parabolic equations by difference methods. I. Linear and quasilinear equations for the infinite interval," Comm. Pure Appl. Math., v. 5, 1952, pp. 155-211. MR 13, 947. 
11. P. D. LAX \& R. D. Richtmyer, "Survey of the stability of linear finite difference equations," Comm. Pure Appl. Math., v. 9, 1956, pp. 267-293. MR 18, 48.

12. R. E. Showalter \& T. W. TING, "Pseudoparabolic partial differential equations," SIAM J. Math. Anal., v. 1, 1970, pp. 1-26.

13. T. W. TING, "Certain non-steady flows of second-order fluids," Arch. Rational Mech. Anal., v. 14, 1963, pp. 1-26. MR 27 \#3224.

14. T. W. TING, "Parabolic and pseudo-parabolic partial differential equations," J. Math. Soc. Japan, v. 21, 1969, pp. 440-453. MR 41 \#8827.

15. R. S. VARga, Matrix Iterative Analysis, Prentice-Hall, Englewood Cliffs, N.J., 1962. MR 28 \#1725.

16. O. B. WIDLUND, "On the stability of parabolic difference schemes," Math. Comp., v. 9, 1965, pp. 1-13. MR 30 \#717. 